\title{
Analysis of second-order effects of constructions behaviour
}

\author{
Raminta Venslavavičiūté ${ }^{1}$, Kęstutis Urbonas ${ }^{2}$ \\ Department of Steel and Composite Structures, Vilnius Gediminas Technical University, Vilnius, Lithuania \\ E-mail: ${ }^{1}$ raminta.venslavaviciute@vgtu.lt (corresponding author)
}

\begin{abstract}
This paper presents the importance of the second-order effects in behaviour of steel frame structures. EN 1993-1-1 were distinguished three main methods: taking / without taking into account second order effects depending on the sensitivity of horizontal design effects and tolerance. Using these methods, the susceptibility of steel frame structures to second-order impacts is considered in accordance with EN 1993-1-1. The study was performed taking into account also the rotational stiffness of the joints of the frame structure. In this way, trying to determine the effect of the joint on the rotational rigidity makes the frame structure more sensitive to second order impacts. It is also considered a semi-rigid joints effects the general frame-tailed P- $\Delta$ and the second row stress. This establishes the link between the effects of the second-order and the semi-rigid joints in the overall (general) analysis. Frame-building sensitivity makes it possible to compare and measure the importance of such dependencies.
\end{abstract}

Keywords: steel frame, structural analysis, second order analysis, semi-rigid joints, finite element method, sensitivity of the second order effects.

\section{Introduction}

Steel structures, compared with the other, is slender, and their design has the greatest impact at the structural strength and its permanence and stability. Slenderness of steel structures are the determining factor for design sensitivity for second-order impacts (Yoo \& Lee, 2011). The second-order effects analysis differs from the first-order by the fact that the deformed shape of the structure, is evaluated. EN 1993-1-1 states that deformed geometry factors (second-order factors) must be taken into account if they significantly increase the load or substantially change the behaviour of the structure. The aim of this research work is to show the close relationship between the second-order analysis and semirigid joints of the $\alpha_{c r}$ coefficient refers to the need to carry out the second-order analysis, where $\alpha_{c r}$-the factor by which the design loading would have to be increased to cause elastic instability in a global mode.

After examining EN 1993-1-1 it is obvious that the code leaves a lot of freedom for the designer himself to decide how to evaluate the effects of the second-order. The analysis of steel frame structures does not detail the methods that properly evaluate the sensitivity of the frame to the second-order effects by introducing semi-rigid joints. Lack of experience in adapting techniques and deviations which causes the misinterpretation of the results. These reasons make it difficult to apply a second-order analysis, so this is not widely used in practice, although it always provides more accurate and safer solutions.

\section{Description of the object}

Construction deforms horizontally until reaching the critical load - it provides the classical theory of Euler. However, depending on the column slenderness, the residual stresses and deviations have a significant effect on the bearing capacity of the column. These factors determine that the structure gradually horizontally deformed before reaching the critical load. This deformation affects the appearance of secondary second-order moments, which further reduces the structural bearing capacity (see Figure 1). In the literature, the second-order global (frame) and local (element) effects are often divided into (frame) P- $\Delta$ and P- $\delta$ (element). P- $\delta$ effects occur along the element, and at the ends of the elements P- $\Delta$ (Ziemian, 2010).

(C) 2019 Authors. Published by VGTU Press. This is an open-access article distributed under the terms of the Creative Commons Attribution (http://creativecommons.org/licenses/by/4.0/) License, which permits unrestricted use, distribution, and reproduction in any medium, provided the original author and source are credited. 

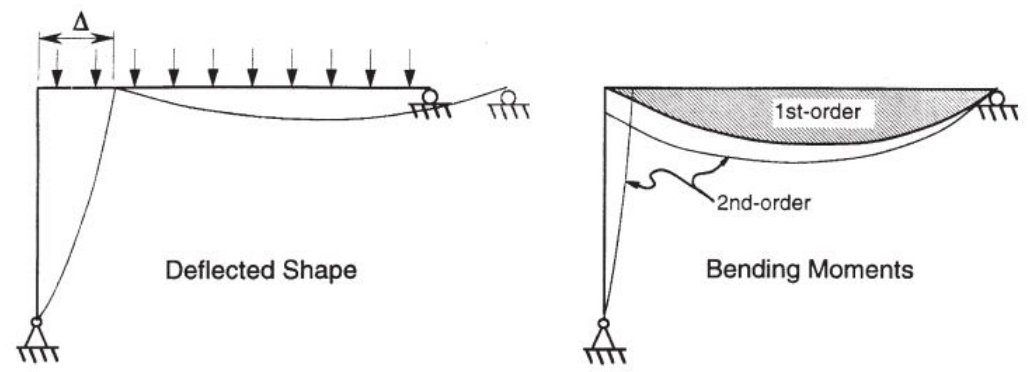

a) sway permitted
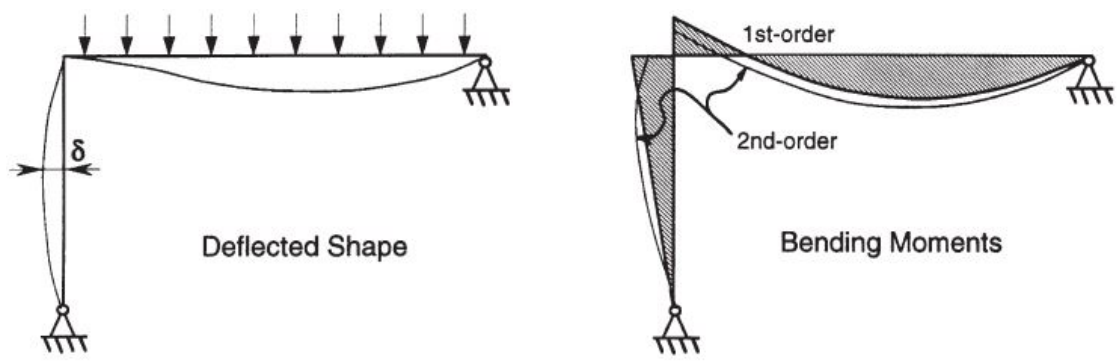

b) sway restrained

Figure 1. Second-order P- $\Delta$ and P- $\delta$ (Ziemian, 2010)

The main purpose of the article is to show the sensitivity of the frame structures for the second-order impacts and the meaning of this $\alpha_{c r}$ coefficient as compared to the full second-order analysis; to study the influence of semi-rigid joints when second-order effects are evaluated. In order to reach the goals it is used the elastic second-order analysis which is permitted in all cases. Therefore, the code provides three main methods for assessing the effects of the secondorder and the deformations according to the type of frame and the chosen type of overall analysis (see EN 1993-1-1 5.2.2(3)):

1. In basic, simple cases - checking the stability of individual elements. Here the buckling length is taken under the common form of buckling;

2. By partially applying an overall analysis, and partly by checking the stability of the individual elements;

3. A detailed second-order analysis is applied.

This article presents the analysis of steel frame structures with different stiffness of joints is considered because joint tendency to deform due to bending moments of the operation are generally critical and has the largest influence on the results obtained in the analysis of structures (Yoo \& Lee, 2011). Frame structure is considered without linking member, although they are often an integral part of frame structures that have a significant effect on their behaviour, but this work does not take into account their influence. Steel frames are only analyzed in a plane of the frame. The analysis of the frame is only in-elastic second-order analysis (analyzing only geometric nonlinearities) is considered (The Steel Construction Institute, 2009).

\section{Analysis of frame}

Sensitivity to second-order effects according to EN 1993-1-1 is determined using the elastic critical load $F_{c r}$ and active force $F_{E d}$ ratio, expressed through the coefficient $\alpha_{c r}$. This coefficient is calculated by using the mentioned equation, the results obtained from the stability analysis, taking into account the original design geometry and common initial inclination.

$$
\alpha_{c r}=\frac{F_{c r}}{F_{E d}},
$$

where: $\alpha_{c r}$-the factor by which the design loading would have to be increased to cause elastic instability in a global mode; $F_{c r}$ - the elastic critical load; $F_{E d}$-active force.

The beams and columns plane type frames may be inspected as restrained horizontal displacements, so the analysis of the first order $\alpha_{c r}$ counted in each floor. Using analytical equation, frames with rigid joints sensitivity of the second order effects: 


$$
\alpha_{c r}=\frac{H_{E d}}{V_{E d}} \cdot \frac{h}{\delta_{H, E d}},
$$

where: $H_{E d}$ - the total design horizontal load transferred by the storey (storey shear); $V_{E d}-$ the total design vertical load on the frame transferred by the storey (storey thrust); $\delta_{H, E d}-$ the horizontal displacement at the top of the storey; $h$ - the storey height.

Different types of structural design joints were considered to be different in the overall analysis (Simões da Silva, Simões, \& Gervásio, 2014). Different analysis has shown that the frame structure with the rigid joints is less sensitive to the second-order effects. Using the results of this frame, the sensitivity of the frame to second-order effects is not useful.

Therefore, during the overall analysis, the focus was on semi-rigid joints, their influence on the resulting displacements and the sensitivity of the frame structure to second-order effects. The results of displacements in the frame with semi-rigid joints is proportional to the stiffness of the joints (see EN 1993-1-8:2005). For multi-storey frames second-order effects may be calculated by increasing the horizontal loads $H_{E d}$ (e.g. wind) and equivalent loads $V_{E d} \varphi$ due to imperfections according to the first order theory by the factor:

$$
\frac{1}{1-\frac{1}{\alpha_{c r}}}
$$

On condition that $\alpha_{c r} \geq 3.0$, where $\alpha_{c r}$ is been calculated according Eq. (2).

The value of the coefficient $\alpha_{c r}$ for determining the sensitivity of frame structures to the second-order effects application of EN 1993-1-1 is only justified if no rigid joints are applied to the frame. For the sake of simplicity, Euronorms allow simplification second-order effects evaluation when $\alpha_{c r}$ value is between 3 and 10 .

Analyzing the selected frame in the second limit state conditions have been previewed, but it was not the primary criterion for assessing the behaviour of the frame structure. The cross sections of the frame were selected based on the load bearing capacity of the element when designing the model for overall analysis, however the requirements of the fitness limit state have not been applied.

\section{Calculations of frame with rigid joints}

Numerical calculations are performed with a multi support two storeys rigid steel frame which is covered by three different loading scheme. The frame is loaded with characteristic permanent load, variable loads: wind, snow and usage, evaluated the emerging horizontal forces that assess the impact of vertical forces on each floor. It involves operating load combinations for computational models are obtained: in a first case of a first combination predominant load is the usage or snow load, and in the second case predominant load is wind. And changing the distribution of the variable load on the floor precisely generates 3 computational load combinations. The general scheme of the object with the layout of the elements is shown in Figure 2.

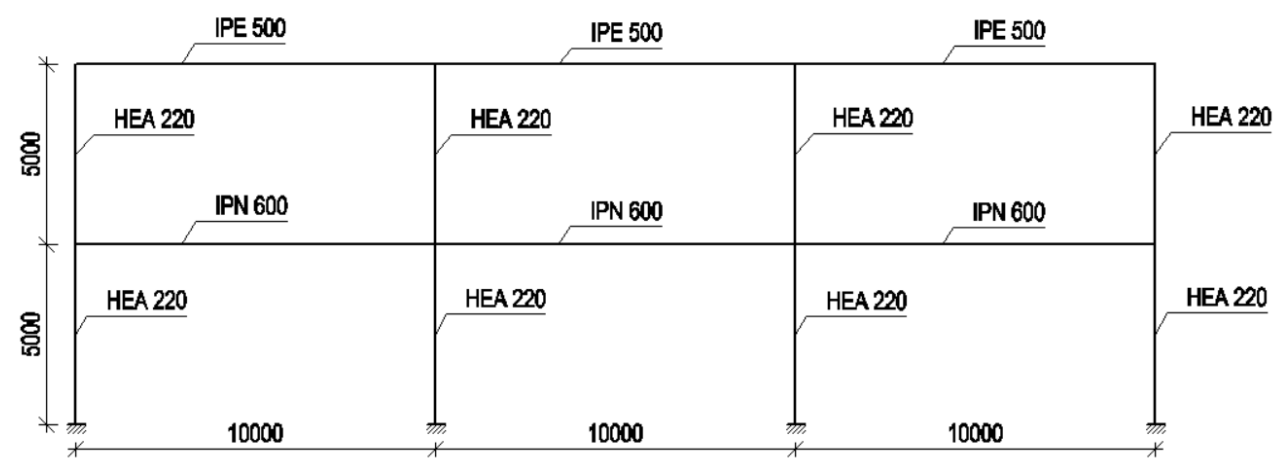

Figure 2. The general scheme of the object with the layout of the elements

Steel frame is calculated using each of three loading schemes to know which version of the load distribution is the most dangerous and the most affective for second-order effects. The actual load situation with pre-set frame columns and beam profiles is analyzed. The expected order of calculation process, when loaded differently arranged on the first floor: started with the variable load evenly distributed over the entire first floor, then a similar loaded uniformly distributed on outside spans and variable load uniformly distributed over the mid-span of the floor. 
The calculations of the steel multi-storey frame performed using different methods of analysis, i.e. the first-order analysis, taking into account the initial geometry of the design and adapting the general initial inclination for the rigid frame. Also, the same frame is calculated applying a semi-rigid joints. Here, the steel frame in question is determined sensitivity second order effects analytically calculating $\alpha_{c r}$ values of each floor. According to the results observed in the steel frame with rigid joints is the least sensitive, so further checks on the calculation $\alpha_{c r}$ validity value is performed with a semi-rigid frame and joints (The Steel Construction Institute, 2009).

Since the steel frame with a semi-rigid joints sensitivity to second order effects evaluating $\alpha_{c r}$ the minimum value is less than 10, during the elastic behaviour analysis must take into account the second-order factors, if they significantly increase the internal forces, or significant changes the behaviour of structures (see EN 1993-1-1:2005). The second-order effects are evaluated using the first-order theory of increased horizontal loads (since the value of $\alpha_{c r}$ is not less than 3) and the second-order analysis of elastic behaviour. The results of the general frame deviations (P- $\Delta$ ) obtained in these two calculations are quite close.

\section{Calculations of frame taking/without taking into account of semi-rigid joints}

According to the combination and load variability, the most sensitive is the frame where variable load evenly distributed over the entire first floor and with semi-rigid joints. Sensitivity is further increased by flexibility of the joints (Daniūnas \& Urbonas, 2010). A rigid frame is not sensitive in any case and requires no further calculations. And frames with semi-rigid joints must be counted in addition to the second-order theory.

$\alpha_{c r}$ is the value received by the lowest value among all floors, in this case, under the first floor.

The results of displacements are represented in the charts below (see Figure 3, Figure 4, Figure 5).

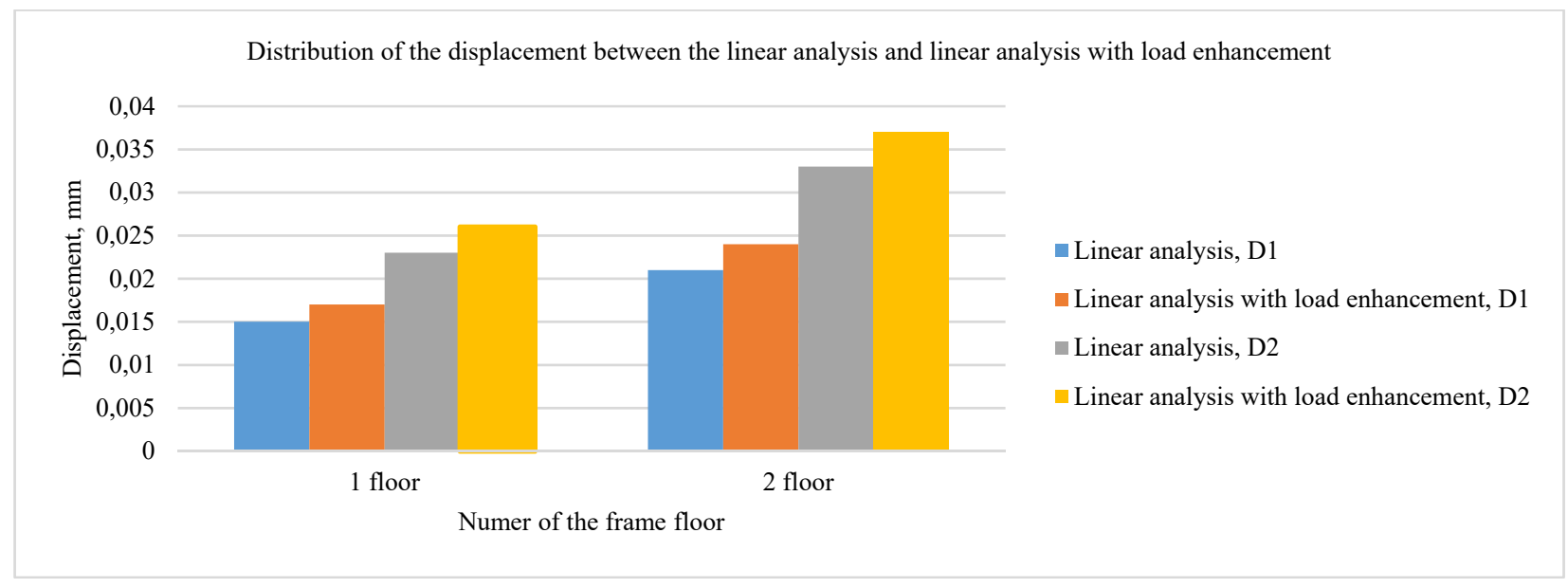

Figure 3. Distribution of the displacement between the linear analysis and linear analysis with load enhancement

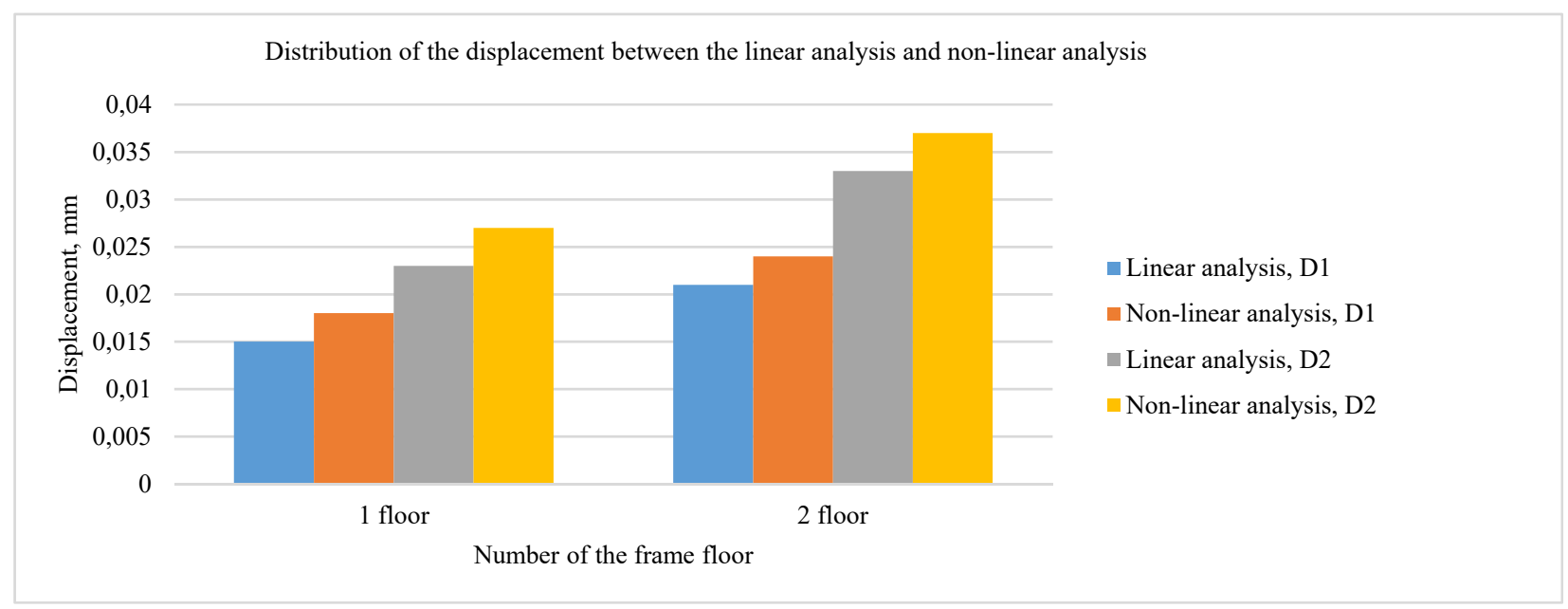

Figure 4. Distribution of the displacement between the linear analysis and non-linear analysis 


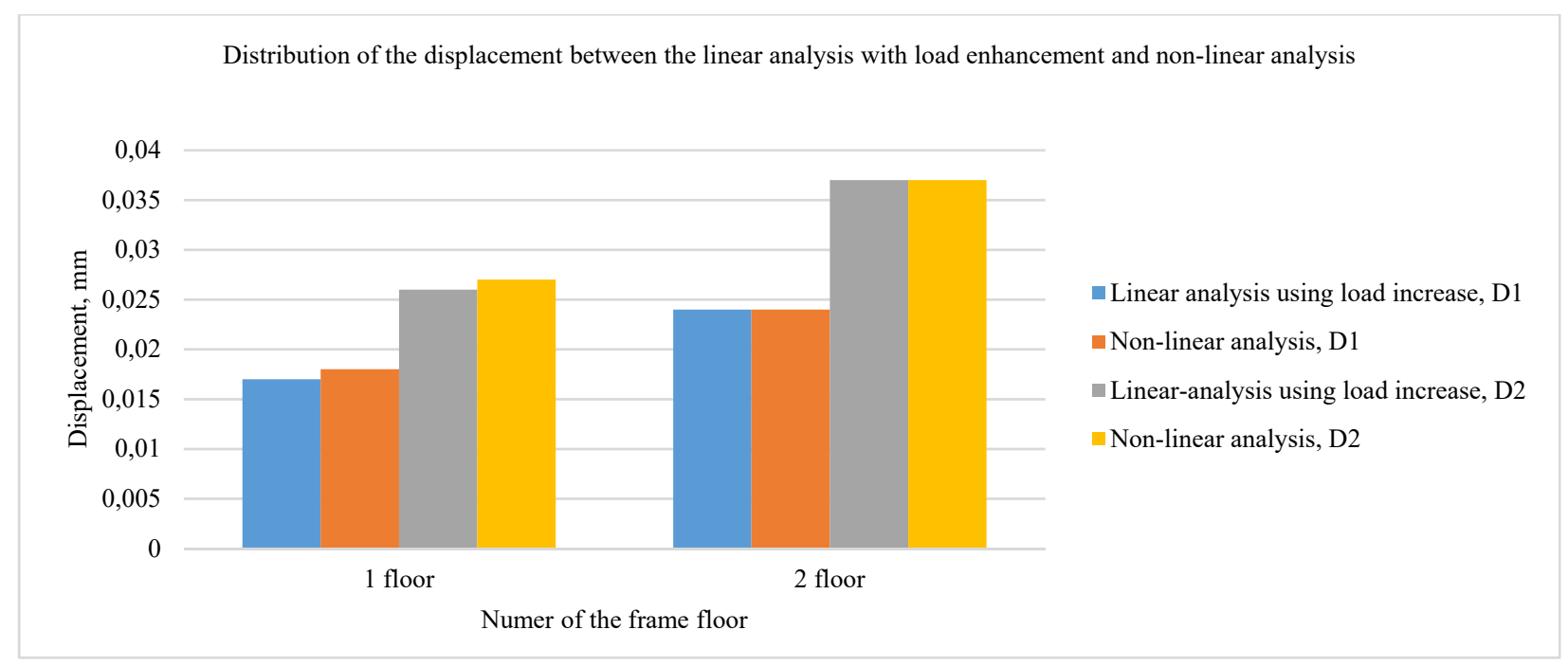

Figure 5. Distribution of the displacement between the linear analysis with load enhancement and non-linear analysis

Meaning of the markings in the chart:

-D1 - first load combination during the calculations;

-D2 - second load combination during the calculations.

After reviewing the results, the main conclusions can be drawn after comparing these results, that the link between the semi-rigid joints and the second-order effects in Euronorm is reasonable and well reflected in the results.

Collected results of the displacements using first-order theory with the load enhancement and the second-order theory, the results are very similar and differ only by $6 \%$.

The greatest influence on the results obtained is not the arrangement of the loads, nor the combinations, but the joints of the frame. Therefore, the more the joint approaches the folding, the greater the displacements and the lower value of $\alpha_{c r}$, this means a growing need for more accurate calculations of performance (see Figure 6).

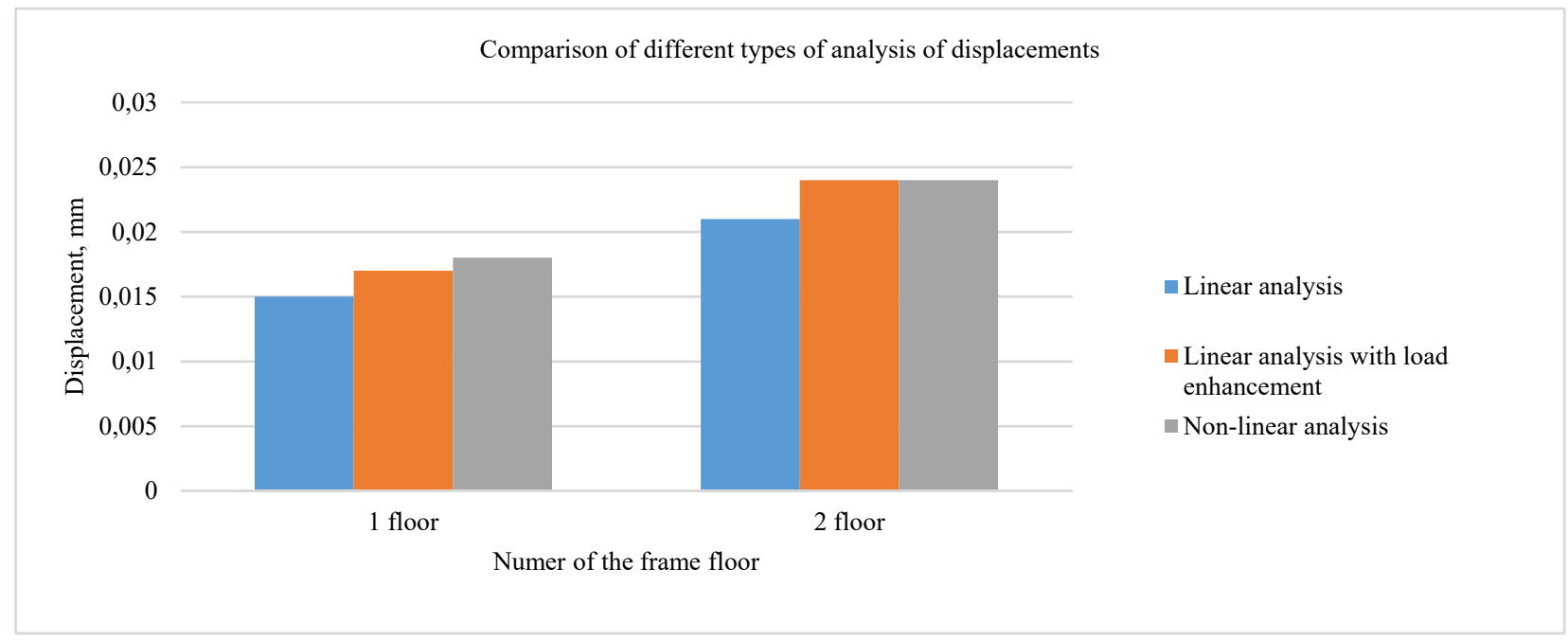

Figure 6. Comparison of different types of analysis of displacements

\section{Conclusions}

The investigatation of the influence of the semi-rigid joints (then second-order effect is taking into account) to the behaviour of the steel frame was made. Also, the sensitivity of the framed structures to second-order effects is checked using $\alpha_{c r}$ coefficient and the meaning of this coefficient compared with a full second-order analysis was evaluated.

Comparing the type of different joints in the frame structure and examining their influence on the second-order effects, it was found that the joints are more flexible, the greater its effect on the second-order effects and on the structure calculation results. 
The comparison of the results was obtained by partially applying an overall analysis and partly by checking the stability of the individual elements, and applying a detailed second-order analysis. It has been found that in both cases the results are close or intuitive (between 3 and 10).

The flexiblility of the joints shows the importance of second-order effects in designing of structures. Because of Euronorms are oriented to design stell structures using semi-rigid joints. Consequently, there appeared necessity for evaluation of geometric nonlinearities.

\section{References}

Brodniansky, J. (2016). Elastic critical buckling mode as imperfection on frame with nonuniform cross-section along the members with imperfection according EN 1993-1-1 clause 5.3.2 (11). In The International Colloquium on Stability and Ductility of Steel Structures - SDSS 2016. Timisoara, Romania.

Daniūnas, A., \& Urbonas, K. (2010). Influence of the semi-rigid bolted steel joints on the frame behaviour. Journal of Civil Engineering and Management, 16(2), 237-241. https://doi.org/10.3846/jcem.2010.27

Simões da Silva, L., Simões, R., \& Gervásio, H. (2014). Design of steel structures (1 ${ }^{\text {st }}$ ed.). Mem Martins, Portugal.

The European Union. (2005). Eurocode 3: Design of steel structures - Part 1-1: General rules and rules for buildings (EN 19931-1:2005).

The European Union. (2005). Eurocode 3: Design of steel structures - Part 1-8: Design of joints (EN 1993-1-8:2005).

The Steel Construction Institute. (2009). Steel building design: Worked examples for students. In accordance with Eurocodes and the UK National Annexes. United Kingdom.

Yoo, C., \& Lee, S. (2011). Stability of structures (1 ${ }^{\text {st }}$ ed.). Burlington, Mass.: Butterworth-Heinemann.

Ziemian, R. (2010). Guide to stability design criteria for metal structures (6 ${ }^{\text {th }}$ ed.). Hoboken, N.J.: John Wiley \& Sons. https://doi.org/10.1002/9780470549087 